\title{
Diagnose it yourself: will there be a home test kit for Alzheimer's disease?
}

\author{
Felix Menne* ${ }^{* 1}$ (i) \& Carola G Schipke ${ }^{1}$ (i) \\ ${ }^{1}$ Predemtec AG, Rudower Chaussee 29, Berlin 12489, Germany \\ *Author for correspondence: Tel.: +49 30403668 882; menne@predemtecdx.com
}

Practice points

- General practitioners are faced with old age patients complaining about decline of cognitive function every day. Timely and accurate diagnosis of neurodegenerative processes potentially leading to dementia is essential for implementing preventive and therapeutic measures.

- The most common cause for dementia is Alzheimer's disease (AD). Today's gold standard for the diagnosis of AD is high in effort and cost. An in vivo diagnosis with $100 \%$ accuracy is still not possible.

- There is a demand for AD testing systems with wide accessibility that give reproducible and reliable results, ideally for use at home.

- Novel techniques are focusing on biomarkers in peripheral specimens, including blood, cells, saliva or urine, yielding conflicting results.

- Thus far, the most promising approach are blood-borne biomarkers targeting analytes usually quantified in cerebrospinal fluid like tau or amyloid. These markers measured in blood can yield reliable and valid results. However, inter- and intra-laboratory variability is still high.

- Other bloodwork approaches are targeting neuroinflammatory and neuroprotective biomarkers, which are known to be involved in neurodegenerative processes such as AD. Another promising marker might be $\mathrm{NfL}$ measured in blood serum or plasma.

- Due to technical limitations, an AD test for sole use at home is unlikely at this point. However, a testing kit for taking blood at home to send directly to a laboratory which in return sends the results with an interpretation, seems technically feasible in the near future.

- Based on the available literature, a panel of APOE genotyping, amyloid-beta 1-42/1-40 ratio, $\mathrm{p}$-tau and NfL quantified in the blood provides a promising biomarker combination.

Alzheimer's disease is the most common neurodegenerative process leading to dementia. To date, there is no curative approach; thus, establishing a diagnosis as early as possible is necessary to implement preventive measures. However, today's gold standard for diagnosing Alzheimer's disease is high in both cost and effort and is not readily available. This defines the need for low-effort and economic alternatives that give patients low-threshold access to testing systems at their general practitioners or even at home for an independent retrieval of a biologic specimen. This perspective gives an overview of established and novel approaches in the field and speculates on the future of test strategies eventually technically implementable at home.

Lay abstract: Alzheimer's disease is a common cause for dementia. While there is no cure yet, finding a diagnosis as early as possible is necessary to slow down worsening of cognitive abilities as much as possible. The commonly administered diagnostic tools for Alzheimer's disease are high in both cost and effort. This emphasizes the need for low-effort and economic alternatives, that give patients a low-threshold access to testing systems at their general practitioners or at home in a self-application. This perspective gives an overview of today's diagnostic standard and reviews novel approaches in the field. It also speculates on the future of strategies that might potentially be suitable for taking a diagnostic test at home.

Tweetable abstract: Will it one day be possible to diagnose Alzheimer's Disease at home? This perspective article is discussing techniques, which might make it feasible in the future.

First draft submitted: 3 December 2020; Accepted for publication: 5 February 2021; Published online: 18 February 2021

Keywords: Alzheimer's disease $\bullet$ biomarkers $\bullet$ dementia $\bullet$ diagnostics $\bullet$ novel techniques 
Millions of people worldwide are affected by neurodegenerative diseases that can lead to dementia [1], first and foremost by Alzheimer's disease (AD). This has large-scale social and economic implications and poses a heavy burden individually for the patients themselves and their caretakers and the whole community in the form of finance needed for diagnostics and treatment of the disease. Thus, there is a need for an easily accessible, minimally invasive, economic and reliable diagnostic test capable of validly detecting the presence of AD earlier than is currently the case. According to a survey conducted by the 2019 World Alzheimer Report [2], 95\% of around 70,000 respondents think they could develop dementia in their lifetime and $82 \%$ would take a genetic test to learn about their risk. Apart from the rare autosomal dominant familial AD, which only accounts for less than $1 \%$ of $\mathrm{AD}$ cases [3], the sporadically inherited $E 4$ variant of $A P O E$ gene has been identified as increasing the risk for $\mathrm{AD}$ [4]. Being aware early of having an elevated risk for developing dementia due to $\mathrm{AD}$ and subsequently making lifestyle changes can delay cognitive worsening in subjects at risk for dementia [5]. There is evidence showing the positive and lasting effect of primary and secondary prevention in $\mathrm{AD}$ [6]. By taking preventive measures, there could be several millions of fewer patients and their caregivers burdened by the disease [7]. Furthermore, accurate diagnostic measures, in general, are in demand not only by the wider public but also by pharmaceutical companies for various other reasons. So far, no causal therapy against $\mathrm{AD}$ is available, but the hunt for adequate curative treatment is continuing. Due to the not fully understood etiology of $\mathrm{AD}$, a wider range of biomarkers might help to determine the individual risk and nature of a patient's disease. This is not only for attaining an easy and economical diagnostic tool identifying participants at risk to include them in clinical trials but also gaining more insight into the causes of the disease to develop a causal treatment eventually.

To understand today's approaches of biomarker-based diagnosis of $\mathrm{AD}$, the currently established hallmarks of $\mathrm{AD}$ need to be considered. The characteristics of $\mathrm{AD}$, the most common cause for dementia, are extracellular amyloid plaques and intracellular neurofibrillary tangles [8]. It is believed that intracerebral cellular changes, especially the accumulation of amyloid, occur up to 20 years before the onset of clinical symptoms, including loss of memory and decline of orientation or speech [9]. The clinical course of AD is usually a slow but gradual process: it was shown that subjective cognitive decline, defined as the subjective experience of worsening cognitive performance among cognitively normal older individuals, can represent an at-risk stage of $\mathrm{AD}[10,11]$. Further down the progress, when clinical symptoms become objectifiable but are not as pronounced as to impair activities of daily living and with neurobiological evidence for underlying $\mathrm{AD}$ pathophysiology, the syndrome of mild cognitive impairment (MCI) due to $\mathrm{AD}$ can be diagnosed [12]. As soon as a patient needs assistance in their daily routines, the clinical stage of dementia is reached.

Reaching the causal underlying alterations for $\mathrm{AD}$ in the brain is hindered by different factors, one important being the blood-brain barrier (BBB). The BBB is a semipermeable border of endothelial cells [13] supposed to protect neurons from pathogens circulating in the blood, while at the same time allowing transport of needed elements like nutrients, glucose or water [14]. However, this highly effective system makes it difficult to gain access to the intracerebral pathological changes and its substrates needed for diagnosing neurodegenerative processes due to the BBB preventing the passage of molecules between the brain and blood system [15].

Despite these challenges, in recent years, there is more and more evidence from peripheral markers that systemic infection and inflammation might play a causal role in AD-related neuropathological processes $[16,17]$. This puts in focus potential biomarkers to measure these changes, where not only a single marker might be relevant but rather a combination of several markers $[18,19]$. In general, it is by now already possible to detect a number of biomarkers in less than $0.5 \mathrm{ml}$ of blood $[19,20]$. Yet, as of now, these analyses require a great amount of effort and time as well as technical equipment and expertise.

In this Perspective article, we give a review on the existing gold standard of diagnosing $\mathrm{AD}$ as well as discuss innovative technologies using specimens such as saliva or peripheral cells. We put a focus on novel techniques that one day might be suitable for use at home, without the necessity of seeing a physician to retrieve a biological specimen for biomarker analysis. Table 1 gives a three-tier graphical overview of the biomarkers evaluated in this article. It recapitulates the cost and effort to analyze the respective markers. Furthermore, we summarize the diagnostic accuracy and speculate on the potential at-home-use. 
Table 1. Graphic evaluation of the potential usability of different novel Alzheimer's disease diagnostic methods.

\begin{tabular}{|c|c|c|c|}
\hline & Analytical cost and effort & Diagnostic accuracy & Potential at-home-use \\
\hline Blood Tau & 0 & + & 0 \\
\hline Blood amyloid & - & + & 0 \\
\hline Blood NfL & 0 & + & 0 \\
\hline Blood neuroinflammatory markers & - & + & 0 \\
\hline Olfactory epithelium & + & 0 & 0 \\
\hline Saliva & 0 & 0 & + \\
\hline Buccal cells & 0 & 0 & + \\
\hline Urine & - & - & + \\
\hline
\end{tabular}

\section{Today's gold standard}

Today, the diagnosis of AD is still complex, even for specialized experts. Depending on the stage of the disease earlier or later - a multitude of information is needed. However, compared with 20 years ago, diagnosing probable $\mathrm{AD}$ has made extensive advances regarding cost and effort. Thanks to those advances, gathering replicable evidence for underlying AD-related neurodegeneration is by now more common.

At specialized centers, neuropsychological assessments and clinical information are the basis to determining different stages of cognitive decline [21,22]. Additionally, there are various methods available to obtain evidence for an underlying $\mathrm{AD}$ pathology in cognitively impaired patients. The more biomarkers and further clinical information are combined, the more accurate the diagnosis [23]. Evidence for AD-typical pathophysiological changes in the brain plays a key role in recent perceptions of a diagnostic strategy: the updated 2018 research framework for the definition of $\mathrm{AD}$ is based on biomarkers [24] and gives a biological definition of the disease. According to this framework, one speaks of $\mathrm{AD}$ when there is evidence for both an abnormal phosphorylated $\mathrm{Tau}(\mathrm{T}+)$ and amyloid-beta 1-42 $(\mathrm{A}+)$ level quantified in cerebrospinal fluid (CSF) or by positron emission tomography (PET) [25,26]. However, both of these methods are solely available in specialized centers. Furthermore, they may be perceived as highly invasive by some patients (lumbar puncture) or patients face a small dose of radiation for a very costly technology (PET). Another important source of information in the diagnosis of AD are brain MRI scans [27]. This is especially needed to assess patterns of brain atrophy and rule out other causes for cognitive decline like vascular damages [28] or brain tumors [29]. While this technology is widely available, it is cost-intensive, up to twice the amount of a computer tomography (CT), which among other disadvantages, lacks the high resolution of MRI scans. Other approaches may add to diagnosing $\mathrm{AD}$, such as EEG or event-related potentials [30] or the examination of eye movement and cognitive processing [31]. Yet again, these techniques require highly specialized personnel and much time per patient and thus are not readily available to the public.

Thus, it is evident that there remains a big gap between the desirable research-based diagnostic measures and the factual community-based diagnostics that outpatients are able to access at their general practitioners or even at home. Furthermore, depending on the number of diagnostic tools used, the diagnostic procedure can be a heavy burden on the patient, their caregivers, and not least on the social system covering the cost.

\section{Blood}

Today, one of the most pursued approaches in the field of biomarkers for AD is the utilization of blood or blood components. Currently, most research activities into blood-borne biomarkers for $\mathrm{AD}$ focus on the biomarkers that were established as gold standard for the diagnosis of AD in CSF: amyloid-beta and Tau species. However, there are continuously evolving approaches to find other markers that might reflect neuropathological processes underlying $\mathrm{AD}$, such as small metabolites. For example, sphingolipids have been found to be associated with measures of progression during preclinical and prodromal $\mathrm{AD}$ and are also discussed as new targets for developing new treatments against the disease $[32,33]$.

\section{Blood - Tau}

While the quantification of total-Tau in the blood may not be the most promising approach [34], the levels of different phospho-Tau species in blood might be more suitable to indicate neurodegenerative processes in the brain. Examining the value of plasma p-tau181 with a newly developed ultrasensitive blood immunoassay, evidence 
shows a gradual increase along the Alzheimer's continuum: young adults and cognitively healthy adults had the lowest concentration, while the highest concentrations were found in amyloid $\beta$-positive MCI and AD groups [35]. Furthermore, plasma p-tau 181 showed a high differential diagnostic ability from brain diseases like frontotemporal or vascular dementia. There is more evidence for the potential diagnostic utility of plasma p-tau181 [36-38] and plasma p-tau217 [39], showing positive associations with PET and CSF tau biomarkers and high diagnostic value to differ between $\mathrm{AD}$ patients, patients with neurodegenerative disorders other than $\mathrm{AD}$ and healthy controls.

\section{Blood - amyloid}

Recent work suggests that quantification of amyloid in blood products might have diagnostic utility. Data suggests that maximally standardized pre-analytical and analytical conditions are the key to reliable measures of amyloid in blood [40,41]. Along this line, data from fully automated Elecsys immunoassays (Roche Diagnostics, Rotkreuz, Switzerland) predict amyloid-beta-positivity (defined by CSF values) with the means of blood plasma amyloidbeta and showed promising results in doing so with AUCs between 0.8 and 0.86 [41]. Also, there is evidence showing that plasma amyloid-beta concentrations measured by mass spectrometry have positive correlations with cerebral $\beta$ amyloidosis [42-44] in the context of AD. Other complex methodological approaches confirm the general suitability of blood amyloid for the diagnosis of $\mathrm{AD}$. The isolation of neuronal-derived exosomes from blood, subsequent transmission electron microscopy and western blot [45] showed results comparable to those for the quantification of CSF biomarkers in subjects at different disease stages. There is even evidence for secondary structure changes in amyloid-beta in blood. It was found that this biomarker when using an immuno-infrared sensor, may detect $\mathrm{AD}$ several years before clinical diagnosis with a positive likelihood ratio of 7.9 [46].

\section{Blood - neuroinflammatory \& neuroprotective markers}

A promising approach outside of the classical markers amyloid and tau in the blood is analyzing levels of neuroinflammatory and neuroprotective serum or plasma protein markers. Since neuroinflammatory pathways become deregulated decades before the clinical onset of $\mathrm{AD}$, targeting these mechanisms might be a novel diagnostic and even therapeutic approach [47]. There is evidence that the combination of the six BDNF, IGF-1, VEGF, TGF- $\beta 1$, MCP-1 and IL-18 quantified in blood serum can indicate the presence of AD at the dementia [19] and [48] MCI stage and can serve as potential diagnostic tools by combining the results with an algorithm. There are more data showing associations between neuroinflammatory markers in serum such as IL-8 and CSF p-tau181 [18] as well as clinical $\mathrm{AD}[49]$.

Blood - NfL

$\mathrm{NfL}$ is an intra-axonal structural protein that is detectable not only in CSF but also in blood plasma and serum and indicates neuroaxonal injury [50]. Plasma (or serum) levels correlate with CSF NfL levels and are increased in both sporadic and familial AD [50]. Plasma NfL is associated with cognitive, biochemical, and imaging features of $\mathrm{AD}$ [36,51]. Furthermore, a rapid increase of NfL is associated with faster worsening in global cognition [52]: plasma NfL levels increased over time in association with baseline CSF biomarkers, in other words, low A $\beta 42$ as well as high total tau and high phosphorylated tau levels.

\section{Olfactory epithelium}

It is well described that olfactory dysfunction increases with the severity of $\mathrm{AD}$ [53], and there is immunohistochemical evidence of tau pathology in the olfactory bulb and nerve that correlates with the severity of AD [54]. There are contradictory reports, but there is evidence for a higher expression of amyloid-beta and paired helical filament tau in the olfactory epithelium of AD patients post mortem in contrast to controls and also to subjects with other neurodegenerative diseases [55]. The methoxy-X04 derivative BSC4090, a fluorescent ligand designed to target neurofibrillary tangles in $\mathrm{AD}$ in olfactory mucosa, might even allow for early differential diagnosis [56]. Thus, smears from the olfactory mucosa might be promising specimens to look into when developing easily applicable approaches for diagnostic implementations.

\section{Saliva}

The examination of saliva components sheds light on a different potential novel biomarker in the detection of AD. The analysis of markers like cortisol [57,58], different metabolites [59,60] or markers of oxidative stress [61,62] yields varying results. However, these findings show that biomarkers outside of amyloid-beta and Tau might be worth 
further investigation. Yet, when analyzing the established AD biomarkers amyloid-beta and Tau, studies in saliva come to different conclusions. Changes in amyloid could be found in different cohorts [63-65], while other studies could not confirm these findings [66,67]. Like in blood, total-Tau alone might not be the most suitable marker in saliva [68], whereas phospho-Tau might improve diagnostic accuracy [66].

\section{Buccal cells}

There is evidence that examining buccal cells for biomarkers in $\mathrm{AD}$ might be a promising approach. Apart from amyloid-beta and Tau, markers of interest in buccal cells are telomeres [69-72], DNA content and structure [73-75] or combined biomarker panels [76], showing the ability to differentiate between healthy control subjects and patients suffering from $\mathrm{MCI}$ and $\mathrm{AD}$. Different studies were able to confirm correlations between buccal cell-derived biomarkers and clinical AD. Furthermore, it was shown that there are correlations between the presence of p-Tau, $\mathrm{t}$-Tau and amyloid-beta in buccal cells with CSF and neuropsychological data $[74,77,78]$. However, as with other novel approaches, results are differing, not least due to lots of different techniques applied and varying biomarkers examined.

\section{Urine}

Promising evidence of urine biomarkers for the diagnosis of $\mathrm{AD}$ in humans is scarce. Two studies found the proteins SPP1, GSN, IGFBP7 [79], and AD7c-NTP levels [80] in urine to significantly differ between AD patients and healthy controls.

\section{Conclusion}

The listed scientific data shows a variety of new approaches that are pursued to facilitate the diagnosis of AD. However, many challenges need to be overcome until any of these approaches can be used for routine diagnostics.

\section{Future perspective}

Since discovering the diagnostic value of amyloid-beta and Tau in CSF, there have been attempts to find biomarkers or signatures indicative of $\mathrm{AD}$ outside of these established ones. Research into these markers has again highlighted that $\mathrm{AD}$ is a disease expressed in very heterogeneous biomarker patterns [81] and clinical features [82]. This complicates finding a singular diagnostic method encompassing this complex process taking place in the brain. It is reasonable to believe that in the future there will be differential diagnoses of $\mathrm{AD}$ subtypes [83], each of which might be defined by alterations in diverse biomarkers - yielding adaptive curative approaches. However, even assuming that various $\mathrm{AD}$ subtypes can be agreed upon, there are various obstacles to overcome until there is a test that can detect $\mathrm{AD}$ in a readily available bodily specimen. From experience gained with the classical CSF biomarkers, a crucial point are the pre-analytical conditions: many studies have shown that a lack of standardization in sampling and processing leads to varying results [84]. Furthermore, different cohorts examined with the same methodology yield different results [85], which yet again illustrates the heterogeneity of the disease and the need for maximal standardization of pre-analytical and analytical conditions. Besides this, there are method-specific challenges that may lead to the measured values not actually mirroring the respective specimen's biomarker level, for example, in immunoassays [86,87].

Thus, the reliability and reproducibility of a potential home test kit is hard to achieve, yet it is needed for enabling a readily interpretable result. Further multicenter studies will need to be conducted to evaluate which method has the lowest inter- and intra-subject variability while at the same time being low in cost and effort. The same holds true for the assessment of inter- and intra-laboratory variability. In these trials, correlations of novel biomarkers will compulsorily need to be undertaken with the established gold standard biomarkers like CSF and PET and in the best case with post-mortem histopathologically confirmed AD cases. At the same time, it needs to be kept in mind that amyloid and Tau in vivo biomarkers only have a specificity and sensitivity of a maximum of $90 \%$ (for amyloid PET) and 53-83\% (for CSF biomarkers), respectively [88]. Also, biomarker levels, especially in neurodegenerative diseases like AD, have to be standardized according to age and potentially sex [89]. For instance, it is a well-replicated finding that CSF tau levels increase naturally with age, without necessarily reflecting a pathological process [90]. This trend could also be shown for NfL, which increases around 3\% per year in CSF of cognitively healthy subjects [91].

Based on the evidence available so far and taking ethical considerations into account, we do not see a home test kit with a dichotomous $\mathrm{AD}$-negative or $\mathrm{AD}$-positive test result in the foreseeable future. From an ethical point of view, the interpretation of medical test results without professional assistance and the potential consequence on a 
patient's well-being is a major challenge, given that health literacy is limited in the general population [92]. However, self-testing kits analyzing genetic information are available, which among other data give information about one's $A P O E$ status, thereby already potentially indicating an increased risk for $\mathrm{AD}$.

Much like already available HIV test kits for at-home-use [93], similarly, in the field of AD we can imagine a testing kit, where a small amount of blood from the fingertip is taken and sent to a laboratory. It would then be processed, and subsequently, the results and an interpretation of the outcome, as well as a recommended course of action are given. Looking at current evidence on the technical feasibility of AD biomarkers, we can see a panel of $A P O E$, amyloid-beta ratio 1-42/1-40, p-Tau and NfL in blood as a promising combination.

However, for the near future, we believe that these results would give information of being at a higher risk for $\mathrm{AD}$ compared with the normal population, rather than a formal diagnosis. With this knowledge, one may implement secondary preventive measures or deliberate with a physician if further diagnostic procedures or treatment is justified. For persons worrying about declining cognitive functioning, we can see this hypothetical test as a method for the exclusion of $\mathrm{AD}$ and thus search for other reasons for cognitive decline in old age, such as vascular etiology [28] or depression [94]. Subsequently, patients and general practitioners may take action to implement according to treatment. For earlier stages of $\mathrm{AD}$, even before the onset of subjective worrying about cognitive decline, there will need to be more research focusing on these subjects before a meaningful test can become available.

Author contributions

F Menne and CG Schipke were responsible for conceptualization, literature research and writing of the manuscript.

Acknowledgments

Thanks to Mr Jack Stewart for proofreading the manuscript.

Financial \& competing interests disclosure

F Menne and CG Schipke are employees of Predemtec AG. This research did not receive any specific grant from funding agencies in the public, commercial or not-for-profit sectors. The authors have no other relevant affiliations or financial involvement with any organization or entity with a financial interest in or financial conflict with the subject matter or materials discussed in the manuscript apart from those disclosed.

No writing assistance was utilized in the production of this manuscript.

Open access

This work is licensed under the Attribution-NonCommercial-NoDerivatives 4.0 Unported License. To view a copy of this license, visit http://creativecommons.org/licenses/by-nc-nd/4.0/

\section{References}

Papers of special note have been highlighted as: $\bullet$ of interest; $\bullet \bullet$ of considerable interest

1. Prince MJ, Wimo A, Guerchet MM, Ali GC, Wu Y-T, Prina M. World Alzheimer Report 2015 - The Global Impact of Dementia: An analysis of prevalence, incidence, cost and trends.. Alzheimers Dis. Int. (ADI) Alzheimer's Disease International, London, UK (2015).

2. Alzheimer's Disease International. World Alzheimer Report 2019: attitudes to dementia. Alzheimers Dis. Int. (ADI) London, UK (2019).

3. Bekris LM, Yu CE, Bird TD, Tsuang DW. Review article: Genetics of Alzheimer disease. J. Geriatr. Psychiatry Neurol. 23(4), 213-227 (2010).

4. Corder EH, Saunders AM, Strittmatter WJ et al. Gene dose of apolipoprotein E type 4 allele and the risk of Alzheimer's disease in late onset families. Science 261(5123), 921-923 (1993).

5. Kivipelto M, Mangialasche F, Ngandu T. Lifestyle interventions to prevent cognitive impairment, dementia and Alzheimer disease. Nat. Rev. Neurol. 14(11), 653-666 (2018).

- Interesting article with data from three randomized clinical intervention trials showing how people with elevated risk of dementia can benefit from multidomain lifestyle interventions.

6. Hsu D, Marshall GA. Primary and secondary prevention trials in Alzheimer disease: looking back, moving forward. Curr. Alzheimer Res. 14(4), 426-440 (2017).

7. Brookmeyer R, Johnson E, Ziegler-Graham K, Arrighi HM. Forecasting the global burden of Alzheimer's disease. Alzheimers Dement 3(3), 186-191 (2007).

8. Blennow K, de Leon MJ, Zetterberg H. Alzheimer's disease. Lancet 368, 387-403 (2006). 
9. Villemagne VL, Burnham S, Bourgeat $\mathrm{P}$ et al. Amyloid $\beta$ deposition, neurodegeneration, and cognitive decline in sporadic Alzheimer's disease: a prospective cohort study. Lancet Neurol. 12(4), 357-367 (2013).

10. Jessen F, Amariglio RE, van Boxtel $\mathrm{M}$ et al. A conceptual framework for research on subjective cognitive decline in preclinical Alzheimer's disease. Alzheimers Dement. 10(6), 844-852 (2014).

-• Groundwork from an initiative of researchers defining the concept of subjective cognitive decline as potential early stage of Alzheimer's disease (AD).

11. Rönnlund M, Sundström A, Adolfsson R, Nilsson LG. Subjective memory impairment in older adults predicts future dementia independent of baseline memory performance: evidence from the Betula prospective cohort study. Alzheimers Dement. 11(11), 1385-1392 (2015).

12. Albert MS, DeKosky ST, Dickson D et al. The diagnosis of mild cognitive impairment due to Alzheimer's disease: Recommendations from the National Institute on Aging-Alzheimer's Association workgroups on diagnostic guidelines for Alzheimer's disease. Alzheimers Dement. 7(3), 270-279 (2011).

13. Daneman R, Prat A. The blood-brain barrier. Cold Spring Harb. Perspect. Biol. 7(1), a020412 (2015).

14. Gupta S, Dhanda S, Sandhir R. Anatomy and physiology of blood-brain barrier. In: Brain Targeted Drug Delivery System - A Focus on Nanotechnology and Nanoparticulates. Elsevier, Amsterdam, The Netherlands, 7-31 (2019).

15. Pardridge WM. CSF, blood-brain barrier, and brain drug delivery. Expert Opin. Drug Deliv. 13(7), 963-975 (2016).

16. Perry VH, Cunningham C, Holmes C. Systemic infections and inflammation affect chronic neurodegeneration. Nat. Rev. Immunol. 7(2), 161-167 (2007).

17. Heneka MT, Carson MJ, Khoury J El et al. Neuroinflammation in Alzheimer's disease. Lancet Neurol. 14(4), 388-405 (2015).

- A comprehensive review on the emerging role of neuroinflammation in $\mathrm{AD}$ and its clinical implications.

18. Popp J, Oikonomidi A, Tautvydaite D et al. Markers of neuroinflammation associated with Alzheimer's disease pathology in older adults. Brain Behav. Immun. 62, 203-211 (2017).

19. Schipke CG, Günter O, Weinert C et al. Definition and quantification of six immune- and neuroregulatory serum proteins in healthy and demented elderly. Neurodegener. Dis. Manag. 9(4), 193-203 (2019).

20. Ouyang M, Tu D, Tong L et al. A review of biosensor technologies for blood biomarkers toward monitoring cardiovascular diseases at the point-of-care. Biosens. Bioelectron. 171, 112621 (2021).

21. Morris JC. Clinical Dementia Rating: a reliable and valid diagnostic and staging measure for dementia of the Alzheimer type. Int. Psychogeriatrics 9(Suppl. 1), 173-176 (1997).

22. Morris JC, Heyman A, Mohs RC et al. The consortium to establish a registry for Alzheimer's disease (CERAD). Part I. Clinical and neuropsychological assessment of Alzheimer's disease. Neurology 39(9), 1159-1165 (1989).

23. Frölich L, Peters O, Lewczuk P et al. Incremental value of biomarker combinations to predict progression of mild cognitive impairment to Alzheimer's dementia. Alzheimers Res. Ther. 9(1), 84 (2017).

24. Jack CR Jr, Bennett DA, Blennow K et al. NIA-AA research framework: toward a biological definition of Alzheimer's disease. Alzheimers Dement. 14(4), 535-562 (2018).

-. The new definition of $\mathrm{AD}$ on a biomarker level set the framework for the next years of research in the field.

25. Müller EG, Edwin TH, Stokke C et al. Amyloid- $\beta$ PET-correlation with cerebrospinal fluid biomarkers and prediction of Alzheimer's disease diagnosis in a memory clinic. PLoS ONE 14(8), e0221365 (2019).

26. Brier MR, Gordon B, Friedrichsen $\mathrm{K}$ et al. Tau and Ab imaging, CSF measures, and cognition in Alzheimer's disease. Sci. Transl. Med. 8(338), 338 ra66 (2016).

27. Johnson KA, Fox NC, Sperling RA, Klunk WE. Brain imaging in Alzheimer disease. Cold Spring Harb. Perspect. Med. 2(4), a006213 (2012).

28. Prins ND, Scheltens P. White matter hyperintensities, cognitive impairment and dementia: an update. Nat. Rev. Neurol. 11(3), 157-165 (2015).

29. Taphoorn MJB, Klein M. Cognitive deficits in adult patients with brain tumours. Lancet Neurol. 3(3), 159-168 (2004).

30. Horvath A, Szucs A, Csukly G, Sakovics A, Stefanics G, Kamondi A. EEG and ERP biomarkers of Alzheimer's disease: a critical review. Front. Biosci. (Landmark Ed.) 23, 183-220 (2018).

31. Freitas Pereira MLG, von Zuben A, Camargo MZ, Aprahamian I, Forlenza OV. Eye movement analysis and cognitive processing: detecting indicators of conversion to Alzheimer's disease. Neuropsychiatr. Dis. Treat. 10, 1273-1285 (2014).

32. Crivelli SM, Giovagnoni C, Visseren L et al. Sphingolipids in Alzheimer's disease, how can we target them? Adv. Drug Deliv. Rev. 159, 214-231 (2020).

33. Varma VR, Oommen AM, Varma S et al. Brain and blood metabolite signatures of pathology and progression in Alzheimer disease: a targeted metabolomics study. PLoS Med. 15(1), e1002482 (2018).

34. Raket LL, Kühnel L, Schmidt E, Blennow K, Zetterberg H, Mattsson-Carlgren N. Utility of plasma neurofilament light and total tau for clinical trials in Alzheimer's disease. Alzheimers Dement. (Amst.) 12(1), e12099 (2020). 
- This work shows the utility of the emerging serum biomarker neurofilament light and its implication for clinical trials in the field of AD.

35. Karikari TK, Pascoal TA, Ashton NJ et al. Blood phosphorylated tau 181 as a biomarker for Alzheimer's disease: a diagnostic performance and prediction modelling study using data from four prospective cohorts. Lancet Neurol. 19(5), 422-433 (2020).

36. Shen X, Li J, Wang H et al. Plasma amyloid, tau, and neurodegeneration biomarker profiles predict Alzheimer's disease pathology and clinical progression in older adults without dementia. Alzheimers Dement. (Amst.) 12(1), e12104 (2020).

37. Park JC, Han SH, Yi D et al. Plasma tau/amyloid- $\beta$ 1-42 ratio predicts brain tau deposition and neurodegeneration in Alzheimer's disease. Brain 142(3), 771-786 (2019).

38. Janelidze S, Mattsson N, Palmqvist $S$ et al. Plasma P-tau181 in Alzheimer's disease: relationship to other biomarkers, differential diagnosis, neuropathology and longitudinal progression to Alzheimer's dementia. Nat. Med. 26(3), 379-386 (2020).

39. Palmqvist $S$, Janelidze S, Quiroz YT et al. Discriminative accuracy of plasma phospho-tau 217 for Alzheimer disease vs other neurodegenerative disorders. JAMA 324(8), 772-781 (2020).

- A promising new approach with the biomarker plasma p-tau-217 and its differential diagnostic utility on which no data was published thus far.

40. Rózga M, Bittner T, Batrla R, Karl J. Preanalytical sample handling recommendations for Alzheimer's disease plasma biomarkers. Alzheimers Dement. (Amst.) 11, 291-300 (2019).

41. Palmqvist S, Janelidze S, Stomrud E et al. Performance of fully automated plasma assays as screening tests for Alzheimer disease-related $\beta$-amyloid status. JAMA Neurol. 76(9), 1060-1069 (2019).

42. Kaneko N, Nakamura A, Washimi Y et al. Novel plasma biomarker surrogating cerebral amyloid deposition. Proc. Jpn. Acad. Ser. B Phys. Biol. Sci. 90(9), 353-364 (2014).

43. Nakamura A, Kaneko N, Villemagne VL et al. High performance plasma amyloid- $\beta$ biomarkers for Alzheimer"s disease. Nature 554(7691), 249-254 (2018).

44. Ovod V, Ramsey KN, Mawuenyega KG et al. Amyloid $\beta$ concentrations and stable isotope labeling kinetics of human plasma specific to central nervous system amyloidosis. Alzheimers Dement. 13(8), 841-849 (2017).

45. Jia L, Qiu Q, Zhang H et al. Concordance between the assessment of Aß42, T-tau, and P-T181-tau in peripheral blood neuronal-derived exosomes and cerebrospinal fluid. Alzheimers Dement. 15, 1071-1080 (2019).

46. Nabers A, Perna L, Lange J et al. Amyloid blood biomarker detects Alzheimer's disease. EMBO Mol. Med. 10, e8763 (2018).

47. Hampel H, Caraci F, Cuello AC et al. A path toward precision medicine for neuroinflammatory mechanisms in Alzheimer's disease. Front. Immunol. 11, 456 (2020).

48. Schipke CG, Menne F, Rubow S, Sigle J-P, Peters O, Grimmer T. Value of a panel of 6 serum biomarkers to differentiate between healthy controls and mild cognitive impairment due to Alzheimer disease. Alzheimer Dis. Assoc. Disord. 34(4), 318-324 (2020).

49. Hesse R, Wahler A, Gummert P et al. Decreased IL-8 levels in CSF and serum of AD patients and negative correlation of MMSE and IL-1ß. BMC Neurol. 16(1), 185 (2016).

50. Khalil M, Teunissen CE, Otto M et al. Neurofilaments as biomarkers in neurological disorders. Nat. Rev. Neurol. 14, 577-589 (2018).

51. Mattsson N, Andreasson U, Zetterberg $\mathrm{H}$ et al. Association of plasma neurofilament light with neurodegeneration in patients with Alzheimer disease. JAMA Neurol. 74(5), 557-566 (2017).

52. Mattsson N, Cullen NC, Andreasson U, Zetterberg H, Blennow K. Association between longitudinal plasma neurofilament light and neurodegeneration in patients with Alzheimer disease. JAMA Neurol. 76(7), 791-799 (2019).

53. Yoo HS, Jeon S, Chung SJ et al. Olfactory dysfunction in Alzheimer's disease- and Lewy body-related cognitive impairment. Alzheimers Dement. 14(10), 1243-1252 (2018).

54. Attems J, Lintner F, Jellinger KA. Olfactory involvement in aging and Alzheimer's disease: an autopsy study. J. Alzheimers Dis. 7(2), 149-157 (2005).

55. Arnold SE, Lee EB, Moberg PJ et al. Olfactory epithelium amyloid- $\beta$ and paired helical filament-tau pathology in Alzheimer disease. Ann. Neurol. 67(4), 462-469 (2010).

56. Pellkofer H, Ihler F, Weiss BG et al. Evaluation of the methoxy-X04 derivative BSC4090 for diagnosis of prodromal and early Alzheimer's disease from bioptic olfactory mucosa. Eur. Arch. Psychiatry Clin. Neurosci. 269(8), 973-984 (2019).

57. Wolf OT, Convit A, Thorn E, De Leon MJ. Salivary cortisol day profiles in elderly with mild cognitive impairment. Psychoneuroendocrinology 27(7), 777-789 (2002).

58. De La Rubia Ortí JE, Castillo SS, Benlloch M, Rochina MJ, Arreche SC, García-Pardo MP. Impact of the relationship of stress and the immune system in the appearance of Alzheimer's disease. J. Alzheimers Dis. 55(3), 899-903 (2017).

59. Yilmaz A, Geddes T, Han B et al. Diagnostic biomarkers of Alzheimer's disease as identified in saliva using $1 \mathrm{H}$ NMR-based metabolomics. J. Alzheimers Dis. 58(2), 355-359 (2017).

60. Tsuruoka M, Hara J, Hirayama A et al. Capillary electrophoresis-mass spectrometry-based metabolome analysis of serum and saliva from neurodegenerative dementia patients. Electrophoresis 34(19), 2865-2872 (2013). 
61. Choromańska M, Klimiuk A, Kostecka-Sochoń P et al. Antioxidant defence, oxidative stress and oxidative damage in saliva, plasma and erythrocytes of dementia patients. Can salivary AGE be a marker of dementia? Int. J. Mol. Sci. 18(10), 2205 (2017).

62. Su H, Gornitsky M, Geng G, Velly AM, Chertkow H, Schipper HM. Diurnal variations in salivary protein carbonyl levels in normal and cognitively impaired human subjects. Age (Omaha) 30(1), 1-9 (2008).

63. Lee M, Guo JP, Kennedy K, Mcgeer EG, McGeer PL. A method for diagnosing Alzheimer's disease based on salivary amyloid- $\beta$ protein 42 levels. J. Alzheimers Dis. 55(3), 1175-1182 (2017).

64. Bermejo-Pareja F, Antequera D, Vargas T, Molina JA, Carro E. Saliva levels of Abeta1-42 as potential biomarker of Alzheimer's disease: a pilot study. BMC Neurol. 10, 108 (2010).

65. Kim C-B, Choi YY, Song WK, Song K-B. Antibody-based magnetic nanoparticle immunoassay for quantification of Alzheimer's disease pathogenic factor. J. Biomed. Opt. 19(5), 051205 (2014).

66. Shi M, Sui YT, Peskind ER et al. Salivary tau species are potential biomarkers of Alzheimer's disease. J. Alzheimers Dis. 27(2), 299-305 (2011).

67. Lau HC, Lee IK, Ko PW et al. Non-invasive screening for Alzheimer's disease by sensing salivary sugar using Drosophila cells expressing gustatory receptor (Gr5a) immobilized on an extended gate ion-sensitive field-effect transistor (EG-ISFET) biosensor. PLoS ONE 10(2), e0117810 (2015).

68. Ashton NJ, Ide M, Schöll M et al. No association of salivary total tau concentration with Alzheimer's disease. Neurobiol. Aging 70, 125-127 (2018).

69. Fenech M, Thomas P. Telomere length in white blood cells, buccal cells and brain tissue and its variation with aging and Alzheimer's disease. Alzheimers Dement. 6(4), S511 (2010).

70. Thomas P, O' Callaghan NJ, Fenech M. Telomere length in white blood cells, buccal cells and brain tissue and its variation with ageing and Alzheimer's disease. Mech. Ageing Dev. 129(4), 183-190 (2008).

71. Mathur S, Glogowska A, McAvoy E et al. Three-dimensional quantitative imaging of telomeres in buccal cells identifies mild, moderate, and severe Alzheimer's disease patients. J. Alzheimers Dis. 39(1), 35-48 (2014).

72. Garcia A, Mathur S, Kalaw MC et al. Quantitative 3D telomeric imaging of buccal cells reveals Alzheimer's disease-specific signatures. J. Alzheimers Dis. 58(1), 139-145 (2017).

73. François M, Leifert W, Hecker $\mathrm{J}$ et al. Altered cytological parameters in buccal cells from individuals with mild cognitive impairment and Alzheimer's disease. Cytom. Part A 85(8), 698-708 (2014).

74. François M, Fenech MF, Thomas $\mathrm{P}$ et al. High content, multi-parameter analyses in buccal cells to identify Alzheimer's disease. Curr. Alzheimer Res. 13(7), 787-799 (2016).

75. Garcia A, Huang D, Righolt A et al. Super-resolution structure of DNA significantly differs in buccal cells of controls and Alzheimer's patients. J. Cell. Physiol. 232(9), 2387-2395 (2017).

76. Leifert WR, Ferdoush Tuli J, Francois $\mathrm{M}$ et al. Buccal cell cytokeratin 14 identifies mild cognitive impairment and Alzheimer's disease in the AIBL study of aging. Curr. Alzheimer Res. 12(3), 233-241 (2015).

77. Hattori H, Matsumoto M, Iwai K et al. The $\tau$ protein of oral epithelium increases in Alzheimer's disease. J. Gerontol. A Biol. Sci. Med. Sci. 57(1), M64-70 (2002).

78. Arredondo LF, Aranda-Romo S, Rodríguez-Leyva I et al. Tau protein in oral mucosa and cognitive state: a cross-sectional study. Front. Neurol. 8, 554 (2017).

79. Yao F, Hong X, Li S et al. Urine based biomarkers for Alzheimer's disease identified through coupling computational and experimental methods. J. Alzheimers Dis. 65(2), 421-431 (2018).

80. Ma L, Chen J, Wang R et al. The level of Alzheimer-associated neuronal thread protein in urine may be an important biomarker of mild cognitive impairment. J. Clin. Neurosci. 22(4), 649-652 (2015).

81. Lukiw WJ, Vergallo A, Lista S, Hampel H, Zhao Y. Biomarkers for Alzheimer's disease (Ad) and the application of precision medicine. J. Pers. Med. 10(3), 138 (2020).

82. Murray ME, Graff-Radford NR, Ross OA, Petersen RC, Duara R, Dickson DW. Neuropathologically defined subtypes of Alzheimer's disease with distinct clinical characteristics: a retrospective study. Lancet Neurol. 10(9), 785-796 (2011).

83. Ferreira D, Nordberg A, Westman E. Biological subtypes of Alzheimer disease: a systematic review and meta-analysis. Neurology $94(10)$, 436-448 (2020).

84. Vos SJB, Visser PJ, Verhey F et al. Variability of CSF Alzheimer's disease biomarkers: implications for clinical practice. PLoS ONE 9(6), e100784 (2014).

- This work shows the importance and the challenges of biomarker processing in the field of AD on the example of cerebrospinal fluid.

85. Mattsson N, Zetterberg H, Janelidze S et al. Plasma tau in Alzheimer disease. Neurology 87(17), 1827-1835 (2016).

86. Bolstad N, Warren DJ, Nustad K. Heterophilic antibody interference in immunometric assays. Best Pract. Res. Clin. Endocrinol. Metab. 27(5), 647-661 (2013). 
87. Sehlin D, Söllvander S, Paulie $S$ et al. Interference from heterophilic antibodies in amyloid- $\beta$ oligomer ELISAs. J. Alzheimers Dis. 21, 1295-1301 (2010)

88. Fink HA, Linskens EJ, Silverman PC et al. Accuracy of biomarker testing for neuropathologically defined Alzheimer disease in older adults with dementia. Ann. Intern. Med. 172(10), 669-677 (2020).

89. Ferretti MT, Iulita MF, Cavedo E et al. Sex differences in Alzheimer disease - the gateway to precision medicine. Nat. Rev. Neurol. 14(8), 457-469 (2018).

90. Sjögren M, Vanderstichele H, Ågren $\mathrm{H}$ et al. Tau and A 342 in cerebrospinal fluid from healthy adults 21-93 years of age: establishment of reference values. Clin. Chem. 47(10), 1776-1781 (2001).

91. Yilmaz A, Blennow K, Hagberg L et al. Neurofilament light chain protein as a marker of neuronal injury: review of its use in HIV-1 infection and reference values for HIV-negative controls. Expert Rev. Mol. Diagn. 17(8), 761-770 (2017).

92. Sørensen K, Pelikan JM, Röthlin F et al. Health literacy in Europe: comparative results of the European health literacy survey (HLS-EU). Eur. J. Public Health 25(6), 1053-1058 (2015).

93. Delaney KP, Branson BM, Uniyal A et al. Performance of an oral fluid rapid HIV-1/2 test: experience from four CDC studies. AIDS 20(12), 1655-1660 (2006).

94. Pantzar A, Atti AR, Fratiglioni L, Fastbom J, Bäckman L, Laukka EJ. Cognitive performance in unipolar old-age depression: a longitudinal study. Int. J. Geriatr. Psychiatry 32(6), 675-684 (2017). 